\title{
FRECUENCIA Y FACTORES DE RIESGO ASOCIADOS A NEUROPATÍA AUTONÓMICA CARDÍACA EN PACIENTES CON DIABETES TIPO 2.
}

\author{
FREQUENCY AND ASSOCIATED RISK FACTORS OF CARDIOVASCULAR AUTONOMIC \\ NEUROPATHY AMONG PATIENTS WITH TYPE 2 DIABETES.
}

\author{
Silvana A Romero ${ }^{1,2}$, Agostina Ortin ${ }^{1}$, Noelia Mercado ${ }^{1}$, Gabriela Caeiro ${ }^{1}$, Jorge Waitman ${ }^{1}$.
}

\section{Resumen:}

Introducción: La neuropatía autonómica cardíaca (NAC) es una complicación frecuente de la diabetes y aumenta la morbimortalidad cardiovascular. A pesar de su prevalencia e impacto está subdiagnosticada. Objetivo: Determinar la frecuencia de NAC y factores de riesgo asociados en pacientes con diabetes tipo 2 (DM2), en el Servicio de Diabetología del Hospital Córdoba. Material y Métodos: estudio transversal, desde Mayo de 2015 a Mayo de 2016. Se realizó Test Autonómicos Cardiovasculares: Maniobra de Valsalva (MV), respiración profunda (RP), razón 30/15 e hipotensión ortostática. Resultados: Se reclutaron 100 pacientes con DM2, 53\% sexo femenino. Edad promedio 52 años, IMC 30,9; HbA1c 8,7\% y evolución de la enfermedad 8,4 años. El 17\% presentaron test normales. El 29\% tenía NAC temprana y $54 \%$ NAC confirmada (10\% NAC severa). Los pacientes con NAC confirmada tenían mayor tiempo de evolución (11 vs 5 años ), mayor duración de insulinoterapia (5 vs 1,5 años ), mayor edad (54 vs 49 años) y mayor riesgo de hipoglucemias severas comparado con pacientes sin NAC o NAC temprana $(p=0,003)$. Factores de riesgo asociados fueron hipertensión arterial OR 2,55 $(\mathrm{p}=0,02)$, neuropatía periférica OR 11,7 $(p<0,0001)$, retinopatía OR 9,03 ( $p=0,001)$, nefropatía OR $3,12(p=0,03)$ y HbA1c $>7 \%$ OR $2,57(p=0,03)$. Conclusiones: Hubo una alta frecuencia de NAC, se asoció con hipertensión, complicaciones microvasculares, mayor edad, duración de la enfermedad, de la insulinoterapia y mal control metabólico. Se debería identificar a pacientes con mayor riesgo, para reducir su aparición e impacto.

Palabras clave: diabetes mellitus; neuropatía diabética; neuropatía autonómica.

\section{Abstract:}

Background: Cardiovascular autonomic neuropathy (CAN) is a common complication of diabetes and is associated with cardiovascular morbidity and mortality. Despite its prevalence, it is usually underdiagnosed. Objective to assess frequency of CAN in type 2 diabetes, treated at the Diabetology Service at Cordoba Hospital, Argentina.

Methods: Cross-sectional study was conducted from May 2015 to May 2016. Cardiovascular Autonomic Tests (Ewing's battery) were performed: response to Valsalva maneuver, expiration-to-inspiration ratio (E/I ratio), standing to lying flat and postural hypotension.

Results: 100 type 2 diabetes patients, $53 \%$ females. The mean age and diabetes duration 52 years old and 8.4 years, respectively. Hemoglobin A1c level of $8.7 \%$ and $47 \%$ were insulin-requiring type 2 diabetes patients. $29 \%$ had early CAN, $54 \%$ confirmed CAN (10\% severe CAN) and $17 \%$ normal cardiovascular tests. Confirmed CAN was higher in those with longer duration of diabetes; longer duration of insulin therapy, older patients and severe hypoglycemia events was higher in those with confirmed CAN versus without CAN or early CAN ( $p$ 0.003). The risk factors associated were hypertension OR 2.55 ( $p 0.02$ ), peripheral neuropathy OR $11.7(p<0.0001)$, diabetic retinopathy OR 9.03 ( 0.001$)$, diabetic nephropathy OR 3.12 ( $p$ 0.03) and hemoglobin A1c > 7\% OR 2.57 ( $p 0,03$ ).

Conclusions: frequency of CAN was high, was associated with hypertension, microvascular complications, older age, poor metabolic control, longer duration of disease and insulin therapy. Patients with a higher risk of developing CAN should be identified, in order to reduce the impact of this complication.

Keywords: diabetes mellitus; diabetes complications, diabetic neuropathy.

1 Servicio de Diabetología, Hospital Córdoba, Córdoba, Argentina.

2 Email de contacto: silvana 136@hotmail.com 


\section{Introducción}

La Diabetes Mellitus (DM) es una epidemia mundial, que afecta aproximadamente 415 millones de personas en el mundo, y se calcula que para el año 2035 esta cifra aumentará a 642 millones ${ }^{1}$. La neuropatía diabética es la complicación crónica más frecuente y afecta a más del $50 \%$ de los pacientes ${ }^{2,3}$. En Argentina, se halló una prevalencia del 34,7\% y las personas con DM tipo 2 (DM2) bajo tratamiento con insulina fueron las más afectadas $(48 \%)^{4}$. Sin embargo, a pesar de su elevada prevalencia y el significativo impacto sobre la calidad de vida de los individuos afectados, sigue siendo subdiagnosticada. La gran variedad de manifestaciones clínicas y la complejidad de los métodos de evaluación contribuyen a la poca identificación de la neuropatía diabética ${ }^{5}$. Esta complicación abarca un grupo de síndromes clínicos y subclínicos que se producen como consecuencia del daño del sistema nervioso somático y/o autonómico ${ }^{2,6,7}$.

La neuropatía autonómica diabética es un desorden común del sistema nervioso autonómico que se desarrolla en pacientes diabéticos o prediabéticos. Entre las neuropatías autonómicas, la neuropatía autonómica cardíaca (NAC) es la forma clínicamente más importante y una de las más estudiadas. Se considera una de las complicaciones más serias de la diabetes y se manifiesta por anormalidades en el control de la variación de la frecuencia cardiaca, así como alteraciones en el control de la tensión arterial ante exigencias fisiológicas ${ }^{2,5}$. La prevalencia reportada de NAC varía ampliamente entre 1,6\% a 90\%, dependiendo de los criterios utilizados para identificarla, el estadio de la enfermedad y la población estudiada ${ }^{8}$. Considerando el estudio poblacional Oxford Community Diabetes Study ${ }^{9}$ y dos amplios estudios europeos multicéntricos, el EURODIAB IDDM Complications Study en personas con diabetes tipo1 $(\mathrm{DM} 1)^{10}$ y el Diacan Multicenter Study Group en pacientes DM1 y DM2 ${ }^{11}$, la prevalencia de NAC en relación a anormalidades en los test cardiovasculares, se estimó entre el 16,8 y 20,9\% en DM1 y entre el 15,8 y el $22,1 \%$ en $\mathrm{DM} 2{ }^{10}$. Si bien la NAC puede estar presente al diagnóstico, la prevalencia incrementa principalmente con la edad, la duración de la diabetes, el control glucémico y las complicaciones crónicas microvasculares ${ }^{4,12-14}$.

La NAC es una complicación común e insidiosa de la DM, asociada a un aumento significativo en la morbilidad y mortalidad cardiovascular, debido al alto riesgo de arritmias cardíacas y muerte súbita, posiblemente relacionado con la isquemia miocárdica silente ${ }^{4,15-17}$. El riesgo relativo de muerte es de casi cuatro veces más frecuente en pacientes con NAC, con un aumento progresivo del riesgo al incrementarse el número de test cardiovasculares alterados ${ }^{18}$. A pesar de su prevalencia y de su impacto clínico y pronóstico, la NAC es habitualmente subdiagnosticada ${ }^{12}$.

La NAC se manifiesta en un amplio espectro de signos y síntomas, como taquicardia en reposo, hipotensión ortostática e intolerancia al ejercicio, sin embargo, estas manifestaciones generalmente no están siempre presentes 8,19 . A diferencia de otros tipos de neuropatía autonómica, la NAC es fácilmente medida en forma ambulatoria. La Asociación Americana de Diabetes (ADA), recomienda que los test cardiovasculares (Test de Ewing) son apropiados para el diagnóstico, porque tienen alta sensibilidad, especificidad, reproducibilidad, y además son fáciles de realizar ${ }^{20}$. En general, se realiza una batería de cuatro pruebas, de las cuales, tres corresponden a respuestas parasimpáticas y una, a respuesta simpática. Éstas consisten en la medición de los espacios R-R en el electrocardiograma (ECG) durante la respiración profunda $(\mathrm{RP})$, maniobra de Valsalva (MV), cambio de decúbito a sentado y test de hipotensión ortostática $^{21}$.

El Grupo de Expertos de Neuropatía Diabética de Toronto, recomienda que el cribado se debe considerar al momento del diagnóstico en DM2, y con más de cinco años de evolución en DM1, particularmente en pacientes con complicaciones microvasculares y macrovasculares ${ }^{22}$.

Dado que su prevalencia no se conoce con exactitud, el diagnóstico es aparentemente sencillo y las consecuencias que acarrea son graves, es importante llevar a cabo una detección exhaustiva para implementar medidas tendientes a reducir su progresión y evitar sus complicaciones.

\section{Objetivos}

Primarios: Determinar la frecuencia de NAC e identificar factores de riesgo asociados a NAC en los pacientes con DM2 en el Servicio de Diabetes del Hospital Córdoba en la Provincia de Córdoba Argentina. 
Secundarios: Establecer la relación entre NAC y la presencia de complicaciones macrovasculares y microvasculares.

\section{Materiales y Métodos}

Se realizó un estudio descriptivo, observacional, transversal en el Servicio de Diabetes de un hospital público, desde el 02 de mayo de 2015 al 02 de mayo de 2016.

Se incluyeron pacientes con DM2 desde el diagnóstico, de ambos sexos, mayores de 18 años, que asisten al consultorio del Servicio de Diabetes del Hospital Córdoba, en la Ciudad de Córdoba. Fueron excluidos pacientes con DM1, con arritmias, marcapaso, retinopatía diabética proliferativa, uso de diuréticos, betabloqueantes y drogas que prolonguen el QT (antipsicóticos, antihistamínicos, macrólidos, cocaína), enfermedades graves (insuficiencia cardíaca clase funcional III-IV de la NYHA ${ }^{23}$, enfermedad pulmonar obstructiva crónica reagudizada, infección, malignidad), pacientes embarazadas, hipoglucemia (menor a $70 \mathrm{mg} / \mathrm{dl}$ ) o hiperglucemia marcada (mayor o igual a $250 \mathrm{mg} / \mathrm{dl}$ ) al momento de realizar la prueba, o que no puedan colaborar en la realización de los test por distintas razones.

Se consignaron las siguientes variables: edad, sexo, tipo de DM, tiempo de evolución de la enfermedad, tratamiento (insulina o antidiabéticos orales), peso, talla, índice de masa corporal (IMC), hipertensión arterial, dislipemia, complicaciones crónicas microvasculares (retinopatía, nefropatía, neuropatía periférica y NAC), complicaciones macrovasculares (accidente cerebrovascular, infarto agudo de miocardio y enfermedad vascular periférica), presencia de hipoglucemia severa en el último año, $\mathrm{HbA}_{1} \mathrm{c}$, glucemia, creatinina, perfil lipídico, excreción urinaria de albúmina y clearence de creatinina calculado por la fórmula Modification of Diet in Renal Disease study (MDRD) ${ }^{24}$.

Los datos se recolectaron a partir de la revisión de la historia clínica, el exámen físico y de la entrevista de cada paciente realizada en el consultorio de diabetes. Los mismos se consignaron en una ficha de recolección de datos.

Para evaluar la presencia de NAC, se realizaron las maniobras de Ewing, recomendadas por la Asociación Italiana para el Estudio del Sistema Neurovegetativo y aprobadas en consenso de la ADA y Academia Americana de Neurología ${ }^{12}$. Durante estas pruebas se realizó un registro continuo de la frecuencia cardíaca con un electrocardiógrafo de un canal digital marca CONTEC modelo CMS80A.

\section{Test Autonómicos Cardiovasculares:}

\section{Test de respiración profunda:}

El test ha sido estandarizado solicitando al paciente que respire profundo, seis respiraciones por minuto, con una inspiración de 5 segundos y espiración de 5 segundos, durante un minuto. La E/l es obtenida calculando la razón entre la media de los 3 intervalos $R R$ más largos durante la espiración y la media de los 3 RR más cortos durante la inspiración.

\section{Razón 30/15 - Test acostado a parado:}

Luego de permanecer en posición supina el individuo es invitado a pararse rápidamente, sin hablar ni moverse hasta el final del estudio (generalmente 30-45 segundos luego de pararse). Puede realizarse ECG y medición de tensión arterial en forma simultánea. El resultado del test es expresado por la razón 30:15, obtenido como la razón entre el intervalo RR más largo entre el 25 y 35 latido luego de pararse y el intervalo RR más corto entre el 10 y 20 latido luego de pararse.

\section{Maniobra de Valsalva:}

La maniobra de Valsalva es una espiración forzada con glotis abierta contra la resistencia. El sujeto en posición de sentado en invitado a soplar con glotis abierta en una boquilla conectada a un manómetro, sin respirar profundo previamente, y manteniendo un esfuerzo espiratorio constante, equivalente a una presión de $40 \mathrm{mmHg}$ por 15 segundos. Luego de ese periodo el sujeto puede respirar regularmente. La variación en la frecuencia cardíaca durante la prueba es obtenida midiendo la razón entre el intervalo $R R$ más largo luego de la espiración y el intervalo RR más corto durante la espiración. 


\section{Test de hipotensión ortostática}

Luego de permanecer el sujeto 5 minutos en reposo supino, se mide la presión arterial en tres o más oportunidades, separadas por un minuto y luego es invitado a pararse rápidamente, y se mide la PA dos veces, luego de 60 y 120 segundos en posición de pie. En la interpretación del test se utiliza la diferencia entre la última PA en posición supina y la más baja durante la posición de pie, considerando hipotensión ortostática $(\mathrm{HO})$ la caída de $30 \mathrm{mmh}$ en la presión sistólica.

Los test de respiración profunda, razón 30/15 y maniobra de Valsalva evalúan la función parasimpática y el test de hipotensión ortostática la función simpática. Para la interpretación de los resultados de estos test, se utilizaron tablas de valores normales ajustadas a la edad del paciente ${ }^{11}$. Se les solicitó que eviten el ejercicio físico intenso 24 horas previas a la prueba cardiovascular, fumar y el consumo de café durante al menos 2 hs antes de realizar el estudio. Todos los participantes firmaron el consentimiento informado, previa evaluación y aprobación por el Comité de Ética del Hospital Córdoba.

Según el Consenso de Toronto sobre $\mathrm{NAC}^{22}$, el diagnóstico y estadificación se define según los siguientes criterios NAC:

$\checkmark$ La presencia de un test cardiovascular alterado es suficiente para el diagnóstico de NAC temprana.

$\checkmark$ La presencia de 2 ó más test cardiovasculares alterados, es necesario para el diagnóstico de NAC definitiva o confirmada.

$\checkmark$ La presencia de hipotensión ortostática, además de los dos criterios anteriores, indica la presencia de NAC severa.

La presencia de retinopatía diabética $(R D)$ se evaluó a través del fondo de ojo, realizado por un oftalmólogo. Los hallazgos son clasificados en: Normal, Retinopatía Diabética No proliferativa (RPDNP) y Retinopatía Diabética Proliferativa (RPDP).

La nefropatía diabética se evaluó según la excreción urinaria de albúmina (EUA) medida en muestras aisladas de orina o en recolecciones de 24 horas. Una EUA $<20$ ugr/min o $<30 \mathrm{mg} / \mathrm{día}$, se clasifica como normoalbuminuria, entre 20-200 ųgr/min o 30-300 mg/día como microalbuminuria y valores $>200$ ųgr/min o $>300 \mathrm{mg} /$ día como proteinuria franca, para lo cuál se requiere por lo menos de dos mediciones mayores a dichos valores, para el diagnóstico de nefropatía diabética ${ }^{25}$.

El diagnóstico de neuropatía diabética periférica se estableció utilizando la valoración de los síntomas y signos mediante el Neuropathy Symptom Scale (NSS) y Neuropathy Disability Score (NDS) en donde se valora la sensibilidad vibratoria, táctil, termoalgésica, fuerza muscular y reflejos osteotendinosos ${ }^{2}$.

Análisis Estadístico: Luego de completar el formulario de datos a partir del análisis de historias clínicas, se realizó un análisis estadístico descriptivo según la naturaleza de las variables, para las mensurables se utilizaron medidas de estadística central y de dispersión (media, desvío standard); y para las de tipo categórico se estudiaron las frecuencias absolutas y relativas porcentuales. Para la ejecución del análisis inferencial se utilizó, en las variables mensurables el test de ANAVA y para el análisis no ajustado de las variables categóricas se utilizó el test Chi Cuadrado y Fisher. Se fijó como nivel de significación p<0,05.

La base de datos inicial fue elaborada en Excel 2010. Para la tabulación y el procesamiento de la información, se utilizó el software para análisis estadístico Infostat versión 2016.

\section{Resultados}

Se reclutaron 100 pacientes con diabetes tipo 2. El 53\% era de sexo femenino. La edad promedio fue de $52 \pm 10$ años, IMC 30,9, HbA1c 8,7 $\pm 2,8 \%$ y una evolución media de la enfermedad de $8,4 \pm 7,2$ años. Del total de los pacientes, $47 \%$ eran insulinorequirientes, con una media de duración de la insulinoterapia de 4 años.

Con respecto a la presencia de factores de riesgo asociados, un 57\% tenía hipertensión arterial, $81 \%$ dislipemia, $36 \%$ sobrepeso y $51 \%$ obesidad. Teniendo en cuenta la presencia de complicaciones crónicas micro y macrovasculares, se presentaron en el $65 \%$ de los pacientes, con la siguiente distribución: $18 \%$ tenían retinopatía, $20 \%$ nefropatía, 52\% neuropatía periférica y $11 \%$ complicaciones macrovasculares. 
TABLA 1: Características basales clínicas y demográficas de la población

\begin{tabular}{|ll|}
\hline & \multicolumn{1}{|c|}{ Total } \\
\hline n & 100 \\
\hline Edad (media \pm DS) años & $52 \pm 10$ años \\
\hline Sexo & $\begin{array}{l}53 \% \text { femenino } \\
47 \% \text { masculino }\end{array}$ \\
\hline IMC (media \pm DS) kg/m2 & $30,9 \pm 6 \mathrm{~kg} / \mathrm{m} 2$ \\
\hline Duración de la enfermedad & $8,4 \pm 7,2$ años \\
\hline Tipos de diabetes & $53 \% \mathrm{DM} 2$ \\
\hline HbA1c (\%) & $47 \% \mathrm{DM} 2 \mathrm{IR}$ \\
\hline Años de insulina & $8,7 \pm 2,8 \%$ \\
\hline Cualquier Complicación crónica & $65 \%$ \\
\hline Retinopatía & $4 \pm 4,5$ años \\
\hline $\begin{array}{l}\text { Nefropatía } \\
\text { Macropatía periférica }\end{array}$ & $18 \%$ \\
\hline HTA DSular & $20 \%$ \\
\hline Dislipemia & $52 \%$ \\
\hline Sobrepeso & $51 \%$ \\
\hline Obesidad & $57 \%$ \\
\hline & $51 \%$ \\
\hline
\end{tabular}

Sólo el $17 \%$ de los pacientes presentaron test autonómicos cardiovasculares normales. La prevalencia general de NAC temprana fue del $29 \%$ y de NAC definida $54 \%$ (de los cuáles $10 \%$ presentaron NAC severa).

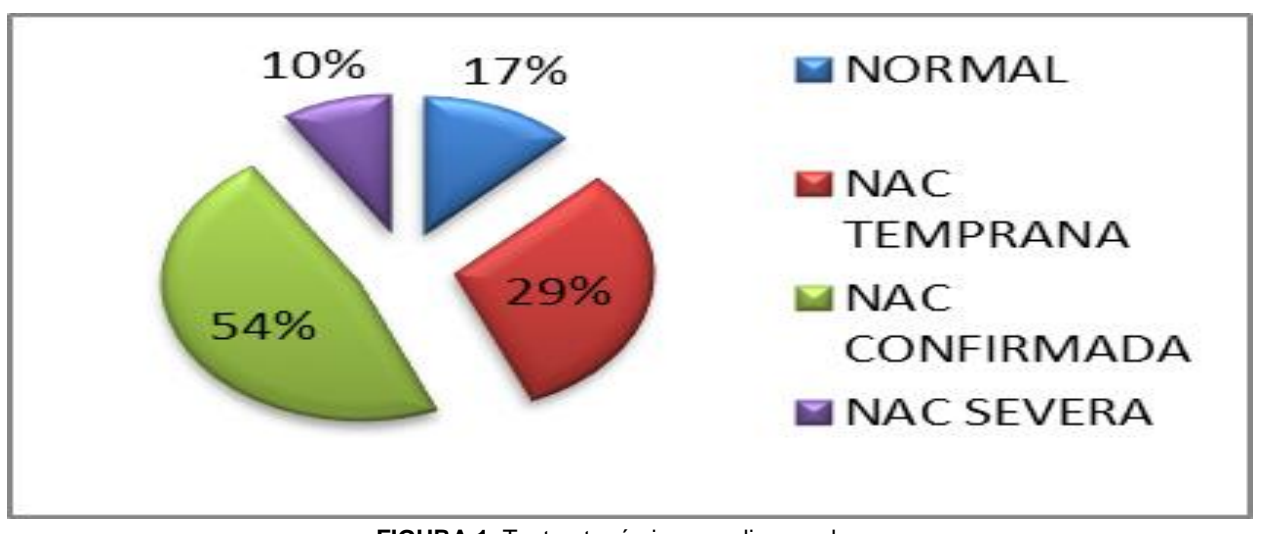

FIGURA 1. Test autonómicos cardiovasculares

Con respecto a los test cardiovasculares realizados, el 53\% de los pacientes tenían una maniobra de Valsalva alterada, el 35\% una maniobra de respiración profunda patológica y el 65\% presentó reducción de la variación de la frecuencia cardíaca con el cambio de posición 


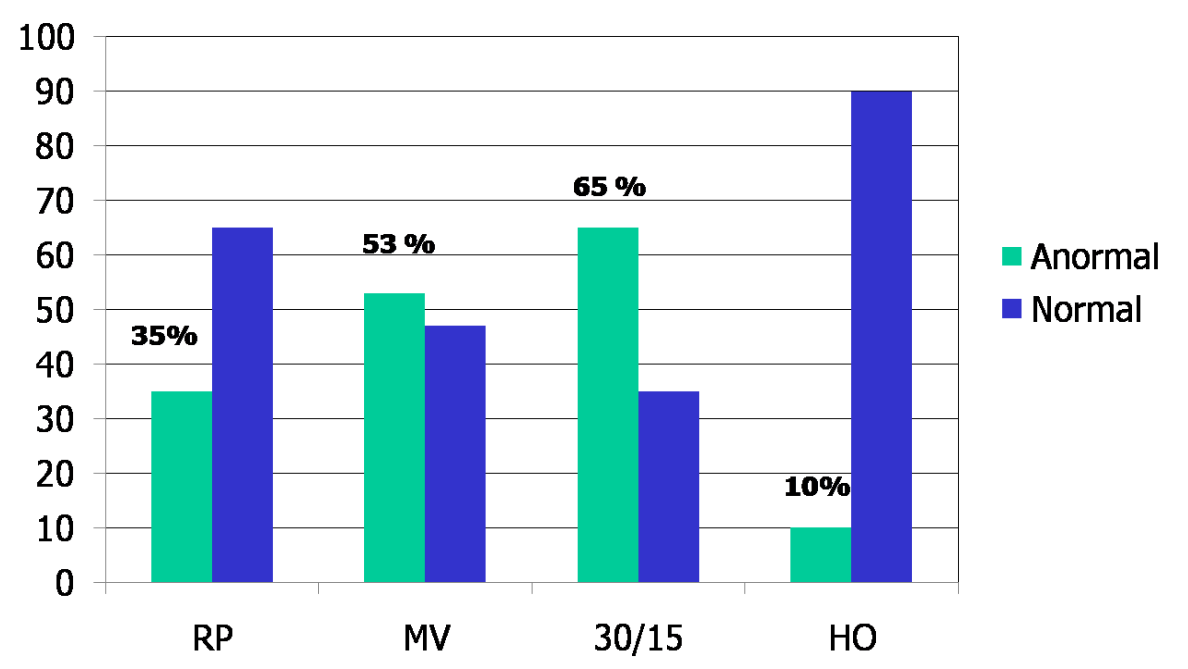

FIGURA 2. Test Autonómicos Cardiovasculares

RP: Respiración profunda, MV: Maniobra de Valsalva, HO: Hipotensión Ortostática.

Los pacientes con NAC confirmada o definitiva tenían mayor tiempo de evolución de la enfermedad (11 vs 5 años $p=0,0001$ ), mayor duración de la insulinoterapia ( 5 vs 1,5 años $p=0,002$ ), mayor edad (54 vs 49 años $p=0,02)$, mayor variación de la frecuencia cardíaca en la maniobra de valsalva $(1,11$ vs 1,32 $p<0,0001$ ), en la maniobra de respiración profunda (10 vs 15 latidos $p<0,02$ ) y en la maniobra de cambio de posición ( 1 vs 1,10 p <0,009) en relación a los pacientes sin NAC o con NAC temprana. Además, los pacientes con NAC confirmada tenían mayor riesgo de presentar hipoglucemias severas en el último año comparado con pacientes sin NAC $(p=0,003)$.

En cuanto a los factores de riesgo asociados a la presencia de NAC confirmada, se encontró una relación directa entre la hipertensión arterial y el riesgo de NAC OR 2,55 (95\% Cl 1,11-5,85; p 0,02), la neuropatía periférica OR $11(95 \% \mathrm{Cl} 4,52-30,51 ; \mathrm{p}<0,0001)$, retinopatía diabética OR 9,03 (95\% Cl 2,20-36,8; $\mathrm{p}$ $0,001)$, nefropatía diabética OR $3,12(95 \% \mathrm{Cl} 1,06-9,20$; p 0,03), y HbA1c >7\% OR 2,57 (95\% Cl 1,06$6,12 ; \mathrm{p} 0,03)$. El tratamiento con insulina se asoció a un mayor riesgo de NAC definida comparado al uso de antidiabéticos orales, OR 6,3 (95\% Cl 1,79-22,74; p 0,004)

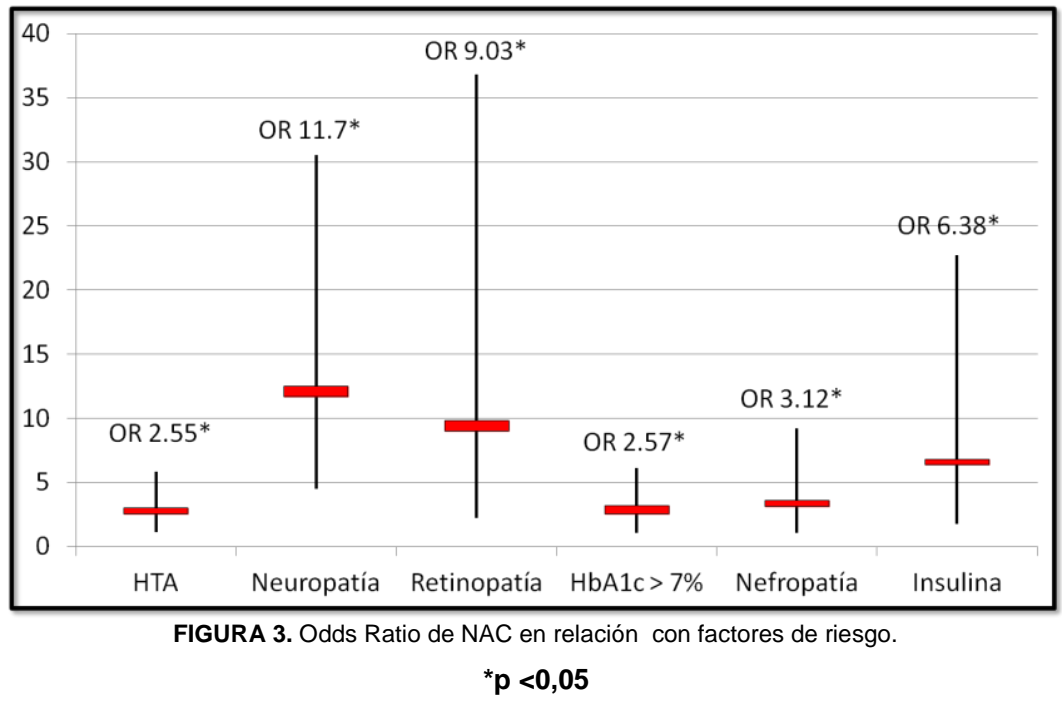

\section{Discusión}

Los informes de prevalencia de NAC varían ampliamente de acuerdo con los métodos de estudio empleados para el diagnóstico y las poblaciones estudiadas. La frecuencia en nuestro trabajo fue elevada y se encontró en un $83 \%$ de los pacientes.

Se encontró una asociación estadísticamente significativa entre la presencia de complicaciones microvasculares con el riesgo de desarrollar NAC. La neuropatía periférica, la retinopatía y la nefropatía diabética aumentaron 12, 3 y 9 veces respectivamente el riesgo de desarrollar NAC. Estos hallazgos 
coinciden con lo reportado en otros estudios, en los cuáles se demostró una relación estrecha entre la existencia de otras complicaciones microvasculares y el desarrollo y severidad de la NAC, debido a que tienen factores de riesgo y una fisiopatología en común ${ }^{4,12-14,26-27}$. A diferencia del estudio publicado por Moususianu y col. ${ }^{28}$, no encontramos asociación con complicaciones macrovasculares, posiblemente por la baja prevalencia de las mismas en la población estudiada (11\%), debido al reducido tamaño de la muestra para evaluar dicha complicación.

En concordancia con otros trabajos ${ }^{13,14}$, encontramos que los pacientes con NAC confirmada tenían de manera estadísticamente significativa mayor tiempo de evolución de la diabetes, edad y mayor duración de la insulinoterapia.

La hipertensión arterial es un predictor de NAC en $\mathrm{DM} 2^{26}$. Además, se han publicado estudios que demuestran que el uso de drogas antihipertensivas (betabloqueantes e inhibidores de la angiotensina II) se asoció con mejoría de la disfunción autonómica, a través del equilibrio simpático-vagal ${ }^{29}$. Consistente con esta evidencia, en nuestro trabajo se demostró que la presencia de hipertensión arterial aumenta 2,5 veces el riesgo de desarrollar NAC.

En DM2, se encontró una correlación significativa entre la modalidad de tratamiento y NAC. Los pacientes tratados con insulina tienen 6 veces más riesgo de desarrollar NAC comparado con el uso de antidiabéticos orales, muy probablemente debido al mal control glucémico previo del inicio de la insulinoterapia. Resultados similares fueron expuestos en estudios previos ${ }^{28}$.

En el estudio EURODIAB ${ }^{30}$ se identificó a la NAC como factor de riesgo independiente para el desarrollo de hipoglucemia severa en pacientes con DM1. El mecanismo sugerido ha sido una disminución en la respuesta contrareguladora frente a la hipoglucemia en pacientes con neuropatía autonómica. Similares hallazgos encontramos en nuestro trabajo. Sin embargo, debido al diseño del estudio, no se puede establecer una relación de causalidad.

A diferencia de los resultados publicados en otros trabajos, no encontramos una asociación estadísticamente significativa entre la presencia de obesidad y NAC, posiblemente debido a que en la población estudiada sólo el 13\% de los pacientes tenían normopeso.

\section{Conclusión}

En conclusión, la NAC es una complicación muy frecuente y de sencilla valoración a través de los test autonómicos cardiovasculares (Test de Ewing). Su frecuencia aumenta con la presencia de hipertensión arterial, complicaciones crónicas microvasculares, con la duración de la enfermedad, edad, mal control metabólico e insulinoterapia.

Consideramos de suma importancia identificar, en base a los hallazgos descriptos en este estudio, a los pacientes con mayor riesgo de desarrollar NAC, para reducir la aparición y el impacto de dicha complicación en la calidad de vida de los pacientes con diabetes.

\section{Bibliografía}

1. Federation ID.IDF Diabetes Atlas - 7Th Edition. Disponible en: URL. http://www.diabetesatlas.org/

2. Comité de Neuropatía Diabética de la Sociedad Argentina de Diabetes. Actualización de Consenso de Neuropatía Diabética- Bahía Blanca, Mayo/ 2009.

3. Boulton A, Malik R, Arezzo J, Sosenko J. Diabetic Somatic Neuropathies. Diabetes Care 2004; $27(6): 1458$.

4. Asociación Latinoamericana de Diabetes. Documentos Selectos de Posición y Consenso de ALAD. 2010.

5. Vinik Al, Maser RE, Mitchell BD, Freeman R. Diabetic autonomic neuropathy. Diabetes Care. 2003; 26(5):155379.

6. Documento de Consenso. Neuropatía diabética. Clasificación, diagnóstico, control metabólico y tratamiento. Rev Soc Arg de Diabetes 1993; 27(3):3-14.

7. Jornadas de Actualización sobre Neuropatía Autonómica Diabética. Rev Soc Arg de Diabetes 2003; 37(3):149158.

8. Akif Serhat Balcıŏlu, Haldun Müderrisoğlu. Diabetes and cardiac autonomic neuropathy: Clinical manifestations, cardiovascular consequences, diagnosis and treatment. World J Diabetes 2015 February 15; 6(1): 80-91.

9. Neil HAW, Thompson AV, John $S$ y col. Diabetic Autonomic neuropathy: the prevalence of impaired heart rate variability in a geographically defined population. Diabet Med 1989; 6:20-24.

10.Stephenson J, Fuller JH. EURODIAB IDDM Complications Study Group. Microvascular and acute complications in IDDM patients: the EURODIAB IDDM Complications Study. Diabetologia 1994; 37: 278-285. 
11.Ziegler D, Gries FA, Mühlen H y col. The DiaCAN Multicenter Study Group, Prevalence and Clinical correlates of cardiovascular autonomic and peripheral diabetic neuropathy in patients attending diabetes centers. Diabetes Metab 1993; 19: 143-151.

12. SpalloneV, Bellavere F, Scionti $L$ y col. Recomendations for the use of cardiovascular test in diagnosing diabetic autonomic neuropathy. On behalf of Diabetic Neuropathy Study Group of the Italian Society of Diabetology Endorsed by the Italian Association for the Study of the Neurovegetative System.

13. Witte DR, Tesfaye $S$, Chaturvedi $N$ y col. EURODIAB prospective complications study group. Risk factors for cardiac autonomic neuropathy in type 1 diabetes mellitus. Diabetologia 2005; 48: 164-7.

14. Ko SH, Park SA, Cho JH, et al. Progression of cardiovascular autonomic dysfunction in patients with type 2 diabetes: a 7-year follow-up study. Diabetes Care 2008; 31:1832-1836.

15. Rodica Pop-Busui, MD. Cardiac Autonomic Neuropathy in Diabetes. A clinical perspective. Diabetes Care, 2010; 33 (2): 434-441.

16. Maser RE, Pfeifer MA, Dorman JS, Kuller LH, Becker DJ, Orchard TJ. Diabetic autonomic neuropathy and cardiovascular risk. Pittsburgh Epidemiology of Diabetes Complications Study III. Arch Intern Med 1990; 150: 1218-1222.

17. Maser RE, Lenhard JM, DeCherney SG. Cardiovascular Autonomic Neuropathy: The Clinical Significance of Its Determination. Endocrinologist 2000; 10: 27.

18. Maser RE, Mitchell BD, Vinik Al y col. The association between cardiovascular autonomic neuropathy and mortality in individuals with diabetes: a meta-analysis. Diabetes Care 2003; 26: 1895-901.

19. Vinik Al, Ziegler D. Diabetic cardiovascular autonomic neuropathy. Circulation 2007; 115:387-397.

20.S. Tesfaye, A. J. M. Boulton, P. J. Dyck et al., "Diabetic neuropathies: Update on definitions, diagnostic criteria, estimation of severity, and treatments," Diabetes Care, vol. 33, no. 12, pp.2285-2293, 2010.

21.M. Jadzinsky, G. Fuente. Diabetes Mellitus.M. Ruiz. Cuarta Edición. 2011. Cap.32, pag 602.

22. Gerasimos Dimitropoulos, Abd a Tahrani, Martin J Stevens Cardiac autonomic neuropathy in patients with diabetes mellitus. World J Diabetes 2014 February 15; 5(1): 17-39.

23. Criteria Committee, New York Heart Association, Inc. Diseases of the Heart and Blood Vessels. Nomenclature and Criteria for diagnosis, 6th edition Boston, Little, Brown and Co. 1964, $p 114$.

24. Levey AS, Coresh J, Greene T, Stevens LA, Zhang YL, Hendriksen S, et al. Using standardized serum creatinine values in the modification of diet in renal disease study equation for estimating glomerular filtration rate. Ann Intern Med. 2006;145(4):247-54.

25. National Kidney Foundation KDOQI Clinical Practice Guidelines and Clinical Practice Recommendations for Diabetes and Chronic Kidney Disease. Am J Kidney Dis 2007;49(Suppl. 2)S12-S154.

26.C. Voulgari, M. Psallas, A. Kokkinos, V. Argiana, N. Katsilambros, and N. Tentolouris, "The association between cardiac autonomic neuropathy with metabolic and other factors in subjects with type 1 and type 2 diabetes," Journal of Diabetes and its Complications, vol. 25, no. 3, pp. 159-167, 2011.

27.P. J. Dyck, J. L. Davies, D. M. Wilson, F. J. Service, L. J.Melton III, and P. C. O'Brien, "Risk factors for severity of diabetic polyneuropathy: intensive longitudinal assessment of the Rochester Diabetic Neuropathy Study cohort," Diabetes Care, vol. 22, no. 9, pp. 1479-1486, 1999.

28. Anca Moususianu, Rodica BslaGa, Septimiu Voidszan, and Zoltán Bajkó. Cardiovascular Autonomic Neuropathy in Context of Other Complications of Type 2 Diabetes Mellitus. BioMed Research International Volume 2013, Article ID 507216.

29. Vinik Al, Murray GL. Autonomic neuropathy is treatable. US Endocrinol 2008; 4:82-84.

30. Stephenson JM, Kempler P, Perin PC, Fuller JH. Is autonomic neuropathy a risk factor for severe hypoglycaemia? The EURODIAB IDDM Complications Study. Diabetologia 1996; 39:1372-1376. 